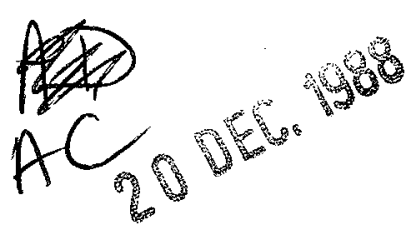

OERN-TIS-RP 219 PP

EUROPEAN ORGANIZATION FOR NUCLEAR RESEARCH

TIS-RP $/ 219 / \mathrm{PP}$

8 December 1988

\title{
HADRON PRODUCTION IN HADRON-NUCLEUS AND NUCLEUS-NUCLEUS COLLISIONS IN A DUAL PARTON MODEL MODIFIED BY A FORMATION ZONE INTRANUCLEAR CASCADE
}

J. Ranft*

ABSTRACT

The concepts of the formation zone and formation zone intranuclear cascade are discussed. The dual parton Monte carlo model is modified by the leading order formation zone cascade. The properties of the resulting model are presented and discussed. It is found that especially rapidity or pseudorapidity distributions change strongly with the value of the formation zone parameter. A comparison with data leads to a phenomenological value of the formation zone parameter of $\tau_{0} c \approx 5$ to $10 \mathrm{fm}$. This corresponds roughly to an average formation zone of $1 \mathrm{fm}$ in the rest frame of the secondary produced.

Submitted to z. Phys. C.

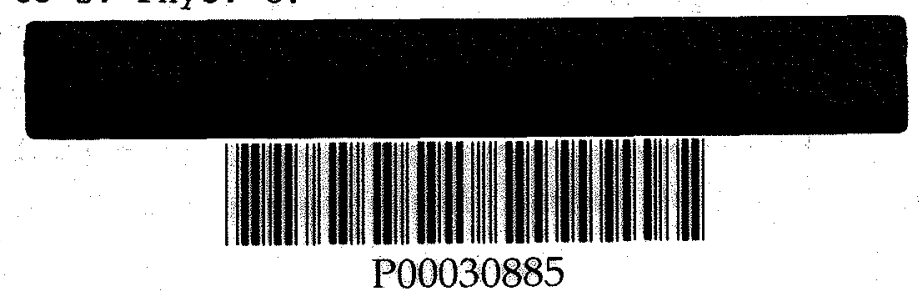

* Permanent address: Sektion Physik, Karl-Marx-Universität, Leipzig. 


\section{INTRODUCTION}

In order to understand which signatures of heavy ion collisions point to the formation of a quark gluon plasma, we should study such collisions within conventional models of hadron physics for soft particle production. Scattering events predicted by such conventional models are the background against which the effects of the quark-gluon plasma have to be found.

The dual parton model was developed by incorporating parton ideas into the dual topological unitarization scheme. It has evolved as a result of the work of many people and it gives a rather complete description of all aspects of soft hadronic multiparticle production in all kinds of high energy collisions.

The main ingredients of the dual parton model are the Regge behaviour of the parton structure functions, fragmentation models for quark-antiquark, quark-diquark and diquark-antidiquart chains and the Glauber multiple scattering theory.

The Monte-Carlo version of the dual multistring fragmentation model has been applied successfully to hadron-hadron collisions [1], hadron-nucleus collisions [2] and nucleus-nucleus collisions [3]. The same models have also been studied using non-Monte-Carlo techniques [4].

In the present paper we discuss the concepts of the formation zone [5] and of the formation zone intranuclear cascade, which was already introduced in leading order into the dual parton model by Ranft [3] and Iga et al. [6]. The properties of the resulting model are studied in order to find out which experimental data offer the best possibilities to determine the formation zone parameter phenomenologically. In comparison with published data we find such a value, but with rather large uncertainties.

In Section 2 we discuss the formation zone and in section 3 the dual parton model as modified by the leading order formation zone cascade. The properties of the particle production in the formation zone dual parton model are discussed in section 4 . In Section 5 we compare with data on transverse energy distributions and pseudorapid- 
ity distributions. In this comparison we consider especially data that include the pseudorapidity region below $n_{1 a b}=2$, which is influenced by the formation zone cascade. In section 6 we present our conclusions and give a phenomenological value for the formation zone parameter. We also point out the need to formulate a model with a full formation zone cascade.

\section{THE FORMATION ZONE}

Landau and Pomeranchuk [5] introduced the formation zone concept while studying the radiation from an electron undergoing multiple scattering when passing through material (see Fig. 1). They found at high energy of the electron a damping of the radiation loss, therefore the electron at high energies becomes more penetrating.

The classical formula for the energy radiated in the form of photons by the electron has the form

$$
\frac{d I}{d^{3} k} \sim\left|\int j(\vec{x}, t) e^{i(\vec{k} \cdot \vec{x}-\omega t)} d^{3} x d t\right|^{2}
$$

This expression can be interpreted as just the inclusive photon distribution. With $\mathrm{dI}=\omega \mathrm{dN}$ we obtain $\mathrm{dI} / \mathrm{d}^{3} \mathrm{k}=\omega \mathrm{dN} / \mathrm{d}^{3} \mathrm{k}$. The amplitude, given by the integral in eq. (1) has to be calculated before squaring. Usually, however, scattering from different atoms does not interfere and it is sufficient to calculate the average radiation emitted in one collision and to calculate dI by multiplying by the number of collisions. This is correct, as long as the exponent in eq. (1) changes rapidly so that collisions at different points have random relative phase factors destroying any interference effects.

Inserting in eq. (1) the current for an electron on a series of straight segments $j \sim m / E \delta(\vec{x}-\vec{v} t)$ the radiation integral becomes

$$
\int_{\text {path }} e^{i(\vec{x} \cdot \vec{v}-1) \omega t} d t
$$

Big or small times between collisions are now measured by the formation zone $\tau$ 


$$
\tau=\frac{1}{\omega(1-\vec{k} \cdot \vec{v})}
$$

By multiplying above and below with the energy of the electron $E$ we obtain

$$
\tau=\frac{E}{k \cdot p}=\frac{E}{m} \cdot \frac{1}{\omega_{e}}
$$

where $\mathrm{k}$ and $\mathrm{p}$ are the 4-momenta of the photon and the electron and $\omega_{\mathrm{e}}$ is the frequency of the photon in the rest frame of the electron. E/m is the time ailation factor from the electron rest frame to the laboratory, and $1 / \omega_{e}$ is the period of the photon. We conclude that the photon cannot be created in a time shorter than its own period. The formation zone is the birth time of the photon as seen in the scattering medium.

The Landau-Pomeranchuk effect is quite important for the energy loss of high energy electrons in matter and it modifies the electronphoton cascades for energies reaching the TeV region [7].

The formation zone concept was first applied by stodolski [8] to hadronic multiparticle production in hadron-nucleus collisions. One uses eq. (4) and translates it to the situation of hadron production. Instead of the radiating electron we consider the projectile hadron with the 4 -momentum $p_{p}=\left(E_{p}, 0.0, \sqrt{E_{p}{ }^{2}-m_{p}{ }^{2}}\right)$ in the laboratory frame, and instead of the photon we consider the secondary hadron with the 4-momentum $p_{s}=\left(E_{s}, \vec{p}_{t s}, \sqrt{E_{s}{ }^{2}-m_{s}{ }^{2}-p_{t s}{ }^{2}}\right)$. This gives for the hadron formation zone in the laboratory frame

$$
\tau_{1 a b}=\frac{2 E_{s}}{\left(m_{p} x\right)^{2}+m_{s}{ }^{2}+p_{t s}{ }^{2}}
$$

Here $x$ is the energy fraction $x=E_{S} / E_{p}$ of the secondary. For most of the produced secondaries the term $m_{p}{ }^{2} x^{2}$ can be neglected. We approximate

$$
\tau_{1 \mathrm{ab}} \approx \gamma_{\mathrm{s}} \tau_{\mathrm{s}}
$$

where $V_{S}=E_{S} / m_{S}$ is the Lorentz factor of the secondary, and

$$
\tau_{s}=\tau_{0} \frac{m_{s}^{2}}{m_{s}^{2}+p_{t s}^{2}}
$$


is the formation zone in the rest frame of the secondary produced. We see that the formation zone in this frame and for $p_{t s} \rightarrow 0$ is just constant.

In the rest of this paper we will use the hadronic formation zone according to eq. (6) and (7).

We use $\tau_{0}$ as an unknown parameter, which has to be determined from experiment.

3. THE FORMATION ZONE INTRANUCLEAR CASCADE IN THE DUAL PARTON MODEL

The Monte-Carlo version of the dual parton model which we will use here has been described in detail in [3]. We refer to this paper and characterize the model here only briefly.

There are altogether $n$ elementary collisions with $n_{p}$ projectile and $n_{t}$ target nucleons involved. The distributions of the number $n, n_{p}$ and $\mathrm{n}_{\mathrm{t}}$ in collisions of the projectile and target nuclei follow from Glauber theory. We follow here the formulation and Monte-Carlo algorithms of Zadorozhnyi et al. [9] (see also [3]).

Central collisions can be defined as collisions where all nucleons of the projectile nucleus interact. (It is assumed that the projectile nucleus is lighter than the target nucleus $A_{p}<A_{t}$.) $A$ rather large fraction of all collisions is central, provided the target nuclei are heavy enough.

The first step in the generation of each Monte-Carlo event is the sampling of the positions of all nucleons in the two colliding nuclei, the sampling of the impact parameter and, using the Glauber theory, sampling the number of elementary collisions $n$ and participating nucleons $n_{p}$ and $n_{t}$.

From the $n, n_{p}$ and $n_{t}$ the chain structure of the event is fixed and we sample next the flavours and momentum fractions $x$ of the valence quarks, diquarks and sea quark-antiquark pairs at the end of the chains. We give also to each multiparticle chain a transverse momentum. 
The next step in the Monte-Carlo calculations is the fragmentation of all chains with masses above the masses of the vector mesons and decuplet baryons. These chains are fragmented using the chain decay code BAMJET [10], which was originally constructed to describe quark-antiquark chains found in hadronic electron-positron events. BAMJET fragments quark-antiquark, quark-diquark and diquark-antidiquark chains into pseudoscalar and vector mesons and octet and decuplet baryons. Subsequently all hadronic resonances decay; this is sampled using the code DECAY [11].

It was shown in [3] that this model describes many features of hadron-nucleus and nucleus-nucleus collisions rather well.

At energies well above $3-5 \mathrm{GeV}$ per nucleon the dual multichain fragmentation model provides a picture of multiparticle production in hadron-nucleus and nucleus-nucleus collisions which is superior to the intranuclear cascade model [12]. In contrast to this, at energies below 3-5 GeV the intranuclear cascade model provides a good description of inelastic nuclear collisions. At these energies the dual multichain fragmentation model - and this is true for all parton models - is difficult to apply.

The physical picture, which explains the absence of the intranuclear cascade at higher energies, is the concept of the formation zone $[5,8]$. Secondary hadrons in the collisions are not formed instantaneously. In their rest frame they need a certain time $t$, which we call the formation time, before they are present as complete hadronic states.

In this picture it appears to be quite natural that most of the fast secondaries created in nuclear collisions have no possibility for secondary interactions inside the nucleus. Because of the relativistic time dilation they are created at positions where the secondary hadronic system is already outside the nucleus. This explains the absence of the intranuclear cascade at high energies and low transverse momenta of the secondaries produced. 
The argument for the absence of secondary interactions inside the nucleus applies only to high energy secondary hadrons, not to particles with low energies and large transverse momenta, which are created inside the target nucleus.

This is also consistent with phenomenological observations. For secondary particles produced in hadron-nucleus collisions with rapidities below $y \approx 2$ in the target rest frame, the model predictions were found to be too low compared with the data $[2,13]$. Otherwise at large transverse momentum one finds an enhanced production (Cronin effect). Starting from these qualitative and phenomenological arguments we propose a leading order correction to slow secondary particle production in asymmetric collisions $\left(A_{p} \ll A_{t}\right)$ in the dual multichain fragmentation model. We introduce an empirical parameter $\tau_{0}$, corresponding to $\tau_{0}$ in formula (7), which we call the hadronic formation time.

It should be noted that in Ref. [3] the formation zone $\tau$ was introduced without the $p_{t}$ dependence given in eq. (7), therefore the parameter $\tau_{0}$ introduced here differs from the formation zone parameter $\tau$ used in Ref. [3]. In fact we expect $\tau_{0}$ to be bigger than the constant $\tau$ used in [3] since for pions with an average transverse momentum $t_{5}$ in (7) will become smaller than $\tau_{0}$.

In the dual Monte-Carlo multichain fragmentation model as formulated above, we know the full space-time history of the collisions. In any particular Lorentz frame we can follow the trajectories of the secondaries created in space and time. We choose in particular the target rest frame, which seems to be the natural frame to describe asymmetric collisions of projectile nucleons or light projectile nuclei with heavy target nuclei. We assume that the secondary hadron starts to interact hadronically in its rest frame after the formation time $\tau_{s}$ or after the time $\tau_{t}=\gamma_{s} \tau_{s}$ in the target rest frame. Assuming a target nucleus with fixed radius $R$ we get the time $t_{R}$, when the secondary particle leaves the target nucleus from

$$
\left(\vec{x}_{0}+\dot{\vec{x}} t_{R}\right)^{2}=R^{2}
$$


A particular secondary particle has the chance to interact again inside the nucleus for $\tau_{t}<t_{R}$. Since we are only interested in the leading order corrections to the multistring fragmentation model we consider such secondary collisions only for hadrons produced in the primary collision. We leave for the future the generalization of this scheme to collisions of heavy projectiles and targets and the construction of a full intranuclear cascade for the soft hadrons.

Besides the primary elementary collisions of the dual chain fragmentation model we have also to sample the events corresponding to secondary interactions of hadrons with nucleons inside the nucleus. These collisions are at rather low energies, often below $1 \mathrm{GeV}$. Therefore it is not practical to sample them using again the multichain fragmentation model. We use instead the code HADRIN [14], which samples inelastic hadron-hadron collisions at energies below 5-9 GeV in good agreement with experiment. HADRIN samples inelastic collisions via quasi two-body reactions and subsequent resonance decay.

4. THE PROPERTIES OF THE DUAL PARTON MODEL WITH FORMATION ZONE CASCADE

In Table 1 we give for some typical collision processes (central and minimum bias) the multiplicities of produced secondary hadrons for formation zones $c \tau_{0}=100$ (this corresponds practically to the model without formation zone cascade), $c \tau_{0}=10$ and $c \tau_{0}=5 \mathrm{fm}$. The average multiplicities, in particular the multiplicities of secondary nucleons, rise with decreasing formation zone. The experimental data, as we will see in Chapter 5, are best described by a formation zone parameter $c \tau_{0}$ between 5 and $10 \mathrm{fm}$. Besides the total average multiplicities we give in Table 1 also the multiplicities of hadrons resulting from secondary interactions in the formation zone intranuclear cascade. We find that for $\tau=5 \mathrm{fm}$ about $50 \%$ of the secondary nucleons are the result of the formation zone cascade.

In Fig. 2 we present pseudorapidity distributions of charged particles and of $\pi^{-}$in central O-Au collisions for the three formation zones $\mathrm{c} \tau=100,10$ and $5 \mathrm{fm}$. The distributions at large pseudorapidity in the laboratory (target) frame is hardly changed by the changing formation zone. The increase of the particle production with decreasing formation zone comes mainly at laboratory pseudorapidities 
below $n=2$. At $n$ around 0 to 1 the production might increase by as much as a factor 2 to 3 . It seems that rapidity or pseudorapidity distributions in the region below $y$ or $n=2$ offer the best possibility to determine the formation zone from experimental data. Also the distributions in the transverse energy will change with the formation zone (see Figs. 10 and 11), but this change is weaker than the one in the rapidity or pseudorapidity distributions.

The formation zone cascade also changes the transverse momentum distribution. The hadrons created by secondary particles interacting in the target nucleus have in general smaller transverse momenta than the interacting particles. This can be seen in Fig. 3, where we plot the ratio of the transverse momentum distribution in 0 -Au collisions with the formation zone $c \tau_{0}=5$ fm to the distribution calculated with the formation zone $c \tau=100$, where no formation zone cascade occurs. The calculated ratio in Fig. 3 can be compared with the transverse momentum ratio

$$
R\left(p_{t}\right)=\frac{\frac{d N}{d p_{t}} \mid 0-A u}{\frac{d N}{d p_{t}} \mid p-p}
$$

as measured experimentally [15]. The comparison suggests that the low $p_{t}$ enhancement in the data might be due to the formation zone cascade. If this is true, then the effect should come in asymmetric collisions $A_{p} \ll A_{t}$ from the rapidity region below $n=2$. It should be easy to check this experimentally.

Next we present some properties of the model which do not depend on the formation zone. In Fig. 4 we compare the multiplicity distribution in the KNO-scaling form as calculated for O-Au collisions at 200 $\mathrm{GeV}$ per nucleon with data from the WA80 experiment given in Ref. [16]. The calculated KNO distribution agrees qualitatively with the data but the number of calculated events is too small for a datailed comparison.

In Fig. 5 we compare the calculated transverse energy distribution in $\mathrm{O}-\mathrm{Pb}$ collisions with data from the NA35 experiment [17]. The data and the calculation refer to the pseudorapidity range $2.2 \leq n \leq 3.8$. The calculated distributions for formation zones $\tau_{0} \mathrm{C}=$ 100, 10 and $5 \mathrm{fm}$ show no significant differences. The formation zone 
cascade has only a minor influence on the distributions in the central region. The agreement of calculated and measured distributions is good.

Fig. 6 gives a similar comparison with data from NA35 [17] in the central region $2.2 \leq n \leq 3.8$ in $\mathrm{p}$-Au collisions at $200 \mathrm{GeV}$. Again there are no significant differences in the calculated distributions obtained with different values of the formation zone parameter $\tau_{0}$.

In Fig. 7 we present a histogram of the total energy of the produced particles in calculated events of $0-W$ collisions. The calculation was performed using the AAEVT code, version 12-88. The quantity plotted is the sum of the total energy of all secondaries produced. Spectator nucleons, which are not involved in the collisions, are not included. The 16 peaks seen result from collisions where 1 to 16 oxygen nucleons take part. The peaks, especially for central collisions, get wider and wider due to the fluctuations in the number of target nucleons which take part in the collision and the subsequent formation zone cascade.

5. COMPARISON WITH DATA TO DETERMINE THE FORMATION ZONE PARAMETER

The distributions which are changed most radically by varying the formation zone parameter are the rapidity and pseudorapidity distributions. This is visible from Fig. 2, and it was already found in [3] when comparing the ratios of the rapidity distributions in $p-X e$ and $\mathrm{p}$-Ar to the ones in $\mathrm{p}-\mathrm{p}$ collisions with experimental data [13].

Here we want to compare to data with the emphasis on the pseudorapidity region below $n=2$. We study simultaneously the pseudorapidity distributions and the transverse energy distributions and extract from the comparison the formation zone parameter $\tau_{0}$. Unforunately, data which are suitable for this task have not been published from the same experiment. The transverse energy distributions measured by the HELIOS collaboration in the range $-0.1 \leq n 2.9$ for $0-A$ collisions [18] and S-A collisions [19] are in the wanted range. But pseudorapidity distributions have not yet been published from this experiment. Such distributions are, however, available from the WA80 experiment [20]. But very often the simultaneous comparison of a model 
with data from different experiments is problematic, especially since one tends to forget the systematical and statistical errors of the experiments and the models.

In Figs. 8 and 9 the model calculations for formation zone parameters $\tau_{0} \mathrm{C}=100,10$ and $5 \mathrm{fm}$ are compared with the data. In the same figures we include also the comparison of the calculated $\pi^{-}$distribution with the measured negative particle distribution of Ref. [21]. The negative particle distribution however changes less with the formation zone parameter than the charged particle distribution which includes the protons from the target.

In Figs. 8 and 9 we compare the model with the same data. In Fig. 9 the agreement is excellent. It is to be noted that the fall of all distributions at the large rapidity end agrees very well with the data. This is the region where the model has hardly any freedom; a change of the formation zone parameter does not change the particle production in this region. In the pseudorapidity region between 0 and 2 the data agrees with the histograms calculated with $\tau_{0} c=5 \mathrm{fm}$.

Unfortunately the same model parameters leading to the good agreement in Fig. 9 lead to transverse energy distributions which extend to larger transverse energies than seen in the data. Therefore the secondary particle multiplicity in the model was reduced to the level of Fig. 8. All other histograms given in this paper are calculated with the model parameters used in Fig. 8. We find in Ref. [20] the remark that the charged particle data might be influenced by the decay of neutral strange particles and that this might have resulted in a $7 \%$ increase of the charged particle multiplicities. We feel that the comparison in Fig. 8 , taking a $7 \%$ reduction of the data into account, might be within the systematical and statistical errors of both the experiment and the Monte Carlo calculation.

Let us point to the model parameters, which allow a change of the multiplicity within, say, $\pm 20 \%$. The masses $M$ of the decaying multiparticle chains in the model follow from the $x$-values $x_{1}$ and $x_{2}$ of the partons at the end of the chains:

$$
\mathrm{M}_{12}^{2}=\mathrm{x}_{1} \mathrm{x}_{2} \mathrm{~s}
$$


The $x$ values have to be selected from the distributions using a certain cut-off to ensure that the masses are large enough for the hadronization. At low energies, where the total energy becomes too small to produce all chains, we have furthermore to reduce the number of chains produced in order to ensure energy-momentum conservation. The cut-off parameters used in this $x$-sampling are to some extent arbitrary and are generally used to get multiplicities in agreement with experimental data.

We return to the comparison with the data. In Figs. 10 and 11 we compare the calculated transverse energy distributions for the formation zone parameters $\tau_{0} c=100,10$ and $5 \mathrm{fm}$ with the HELIOS data measured in $\mathrm{O}-\mathrm{W}$ [18] and $\mathrm{S}-\mathrm{W}$ [19] collisions. The best agreement with the data is obtained for formation zone parameters $\tau_{0} C \approx 5-10 \mathrm{fm}$. The histograms calculated are the result of 600-1000 events only. With this statistics it is not possible to extract the formation zone parametex with smaller errors from this comparison. A more meaningful comparison should also include the trigger conditions and efficiencies of the experiments.

\section{SUMMARY AND CONCLUSIONS}

We have studied the influence of a limited formation zone cascade on particle production on nuclear targets. It is found that this cascade strongly influences the particle production at laboratory rapidities or pseudorapidities below $n=2$. This makes it possible to extract a phenomenological value of the formation zone parameter $\tau_{0}$ comparing the Monte Carlo model to experimental data.

The approach used in the present paper is only a first step in studying the formation zone cascade. The Monte Carlo model should be improved in two ways:

i) By treating the cascading of the produced particles in the projectile as well as in the target. This would then allow to study also reactions like sulfur on sulfur or collisions of projectiles heavier than sulfur. 
ii) By treating the formation zone intranuclear cascade completely, not only the interaction of the first generation secondaries.

Work to implement both aspects has started, but this will result in a Monte Carlo program far more complex than the present one.

From the comparison with data on pseudorapidity and transverse energy distributions we find a best value of the formation zone parameter $\tau_{0} c \approx 5$ to $10 \mathrm{fm}$. This corresponds to average formation zones of roughly $1 \mathrm{fm}$.

\section{ACKNOWLEDGEMENTS}

The author thanks Dr. K. Goebel at CERN and Dr. S. Kahana at BNL for the possibility to perform the calculations reported during stays in these Laboratories. He acknowledges furthermore useful discussions with I. Derado, H.H. Gutbrod, H.J. Möhring, H.R. Schmidt and J. Schukraft. 
REFERENCES

1. J. Ranft and S. Ritter, Z. Phys. C27, 413 (1985); J. Ranft, Z. Phys. C33, 517 (1987); P. Aurence, F.W. Bopp and J. Ranft, Z. Phys. C23, 67; P. Aurenche, F.W. Bopp and J. Ranft, Phys. Rev. D33, 1867 (1986).

2. J. Ranft and S. Ritter, Z. Phys. C20, 347 (1983); J. Ranft and S. Ritter, Z. Phys. C27, 569 (1985).

3. J. Ranft, Phys. Rev. D37, 1842 (1988); H.J. Móhring, J. Ranft and S. Ritter, Z. Phys. C27, 419 (1985); J. Ranft, Phys. Lett. 188B, 379 (1987).

4. A. Capella and J. Tran Thanh Van, Phys. Lett. 93B, 146 (1980): Nucl. Phys. A 461, 501C (1987); A.B. Kaidalov and K.A. TerMartirosyan, Sov. J. Nucl. Phys. 39, 979 (1984); A.B. Kaidalov, K.A. Ter-Martirosyan and Yu. M. Shabelskii, Yad. Fiz. $\underline{43}, 1282$ (1986); A. Capella, C. Pajares and A.V. Ramallo, Nucl. Phys. B, 241, $75(1984)$.

5. L. Landau and I. Pomeranchuk, Dokl. Akad. Nauk SSR, 92, 535 $(1953) ; 92,734$ (1953).

6. Y. Iga, R. Hamatsu, S. Yamazaki and H. Sumiyoshi, Z. Phys. C38, $557(1988)$.

7. T. Stanev, Ch. Vaukov, R.E. Streitmatter, R.W. Ellsworth and T. Bowen, Phys. Rev. D25, 1291 (1982).

8. L. Stodolski, Proc. Vth Int. Colloquium on Multiparticle Reactions, Oxford, 1975, p. 577.

9. A.M. Zadorozhnyi, W.W. Uzhinskii and S. Yu. Shmakov, Dubna preprint P2-86-361 (1986) unpublished. 
10. S. Ritter and J. Ranft, Acta Phys. Pol. B11, 259 (1980);

S. Ritter, Z. Phys. C16, 27 (1982; S. Ritter, Comput. Phys. Commun. $31,393(1984)$.

11. K. Hänßgen and S. Ritter, Comput. Phys. Commun. 31, 411 (1984).

12. H.W. Bertini, Phys. Rev. 137, 1801 (1963); H.W. Bertini, Phys. Rev. 188, 1711 (1969).

13. C. De Marzo et al., Phys. Rev. D26, 1019 (1982).

14. K. Hänßgen and J. Ranft, Nucl. Sci. Eng. 88, 537 (1984).

15. R. Stock, Minirapporteur talk at the XXIV Int. Conf. on High Energy Physics, Munich (1988).

16. J. Schukraft, Talk at the 19th Symp. on Multiparticle Dynamics, Arles, June 1988, preprint CERN-EP/88-141 (1988).

17. A. Bamberger et al., NA35 Collaboration, Phys. Lett. B 184, 271 $(1987)$.

18. T. Åkesson et al., HELIOS collaboration, z. Phys. C 38, 383 $(1988)$.

19. T. Åkesson et al., HELIOS collaboration, CERN preprint CERNEP/88-121 (1988).

20. R. Albrecht et al., WA-80 Collaboration, Phys. Lett. B 202, 597 $(1988)$.

21. A. Bamberger et al., Z. Phys. C $\underline{38}, 89$ (1988). 
Table 1

Average multiplicities calculated by AAEVT in hadron-nucleus and nucleus-nucleus collitions. The numbers in brackets give the multiplicities of particles resulting from the formation zone intranuclear cascade

\begin{tabular}{|c|c|c|c|c|c|c|c|c|c|}
\hline Reaction & $\begin{array}{c}\tau_{o} \\
(f m)\end{array}$ & $\begin{array}{c}\text { Energy } \\
(\mathrm{GeV} / \mathrm{Ap})\end{array}$ & \multicolumn{2}{|c|}{${ }^{n}$ tot } & \multicolumn{2}{|c|}{$\mathrm{n}_{\mathrm{ch}}$} & \multicolumn{2}{|c|}{$\mathrm{n}_{\mathrm{p}}$} & $\mathrm{n}_{\pi^{-}}$ \\
\hline$O-W$ & 100 & 200 & 566 & $(0)$ & 318 & $(0)$ & 27.6 & $(0)$ & 133 \\
\hline \multirow[t]{2}{*}{ central } & 10 & & 602 & $(77)$ & 338 & $(45)$ & 41.1 & $(15.5)$ & $137(15.4)$ \\
\hline & 5 & & 673 & $(198)$ & 379 & $(116)$ & 65.0 & $(41.1)$ & $145(37.8)$ \\
\hline $0-A G$ & 100 & 200 & 500 & $(0)$ & 281 & $(0)$ & 23.4 & $(0)$ & 117 \\
\hline \multirow[t]{2}{*}{ central } & 10 & & 525 & $(43.6)$ & 296 & $(25.0)$ & 31.3 & $(8.6)$ & $(8.4)$ \\
\hline & 5 & & 570 & $(126)$ & 321 & $(73.7)$ & 48.0 & $(26.1)$ & $125(24.1)$ \\
\hline \multirow[t]{3}{*}{$S-W$} & 100 & 200 & 354 & $(0)$ & 198 & $(0)$ & 18.2 & $(0)$ & 82.3 \\
\hline & 10 & & 412 & $(42)$ & 231 & $(24)$ & 28.0 & $(8.2)$ & $92.9(8.2)$ \\
\hline & 5 & & 426 & $(101)$ & 240 & (59) & 38.6 & $(21)$ & 92.5 \\
\hline \multirow[t]{3}{*}{$O-W$} & 100 & 200 & 234 & $(0)$ & 132 & $(0)$ & 12.3 & $(0)$ & 54.6 \\
\hline & 10 & & 240 & $(24)$ & 136 & $(15)$ & 17.0 & $(5.3)$ & $54.2(5.1)$ \\
\hline & 5 & & 286 & $(72)$ & 161 & $(42)$ & 26.7 & $(15.1)$ & $61.6) 13.3$ \\
\hline \multirow[t]{3}{*}{$\mathrm{p}-\mathrm{Au}$} & 100 & 200 & 35.5 & $(0)$ & 20.1 & $(0)$ & 2.0 & $(0)$ & $(0)$ \\
\hline & 10 & & 39.3 & $(7.0)$ & 22.1 & $(3.2)$ & 3.1 & $(1.2$ & $(1.1)$ \\
\hline & 5 & & 43.1 & $(14.5)$ & 24.3 & $(7.5)$ & 4.5 & $(2.7)$ & $(2.4)$ \\
\hline
\end{tabular}




\section{FIGURE CAPTIONS}

Fig. 1 Bremsstrahlung of an electron undergoing multiple scattering.

Fig. 2 Pseudorapidity distributions for charged secondaries and $\pi^{-}$ mesons created in O-Au collisions as calculated in the dual parton model with three different formation zones, $\tau_{0} c=100$, 10 and $5 \mathrm{fm}$.

Fig. 3 The ratio of the inclusive transverse momentum distribution in p-Au collisions as calculated in the model with formation zone cascade $\left(\tau_{0} \mathrm{C}=5 \mathrm{fm}\right)$ to the model without formation zone cascade. This ratio is compared with the ratio of the measured transverse momentum distributions in O-Au collisions to the one in $\mathrm{p}-\mathrm{p}$ collisions $[15]$.

Fig. 4 The KNO multiplicity distribution as calculated for O-Au collisions with $\tau_{0} \mathrm{c}=5 \mathrm{fm}$ compared with data from the WA80 experiment $[16]$.

Fig. 5 Transverse energy distributions calculated for $\mathrm{O}-\mathrm{Pb}$ collisions in the pseudorapidity range $2.2 \leq n \leq 3.8$ for formation zones $c \tau_{0}=100,10$ and $5 \mathrm{fm}$. The calculated distributions are compared with data in the same pseudorapidity range from NA35 experiment [17].

Fig. 6 Transverse energy distributions calculated for $p$-Au collisions at $200 \mathrm{GeV}$ in the pseudorapidity region $2.2 \leq n \leq 3.8$ and for formation zone parameters $\mathrm{c} \tau_{0}=100,10$ and $5 \mathrm{fm}$. The calculated distributions are compared with data from the NA35 experiment [17].

Fig. 7 Event energy distribution in $0-W$ collisions. The energies plotted are the sums of the total energies of all secondaries (this includes the rest energies of the target and projectile nucleons which take part in the collision) produced in the calculated events. Non-participating spectator nucleons are not included. The peaks correspond to collisions with 1 to 16 participating projectile nucleons. 
Fig. 8 Comparison of calculated pseudorapidity distributions for charged particles and $\pi^{-}$mesons produced in O-Au collisions with formation zone parameters $\tau_{0} \mathrm{C}=100,10$ and $5 \mathrm{fm}$ with data of the WA-80 Collaboration [20] and the NA-35 Collaboration [21]. The calculation was done using the same model parameters as in all other figures (except Fig. 9) of this paper.

Fig. 9 See Fig. 8. The calculation here was done using model parameters which did not lead to agreement with the transverse energy distribution presented in Figs. 5, 10 and 11 .

Fig. 10 Comparison of calculated transverse energy distributions in $0-W$ collisions with data measured in the pseudorapidity range $-0.1 \leq n \leq 2.9$ [18]. The calculations are for the formation zone parameters $\tau_{0} c=100 \mathrm{fm}(\mathrm{a}), 10 \mathrm{fm}$ (b) and $5 \mathrm{fm}$ (c).

Fig. 11 Comparison of calculated transverse energy distributions in S-W collisions with data measured in the pseudorapidity range $-0.1 \leq n \leq 2.9$ [19]. The calculations are for the formation zone parameters $\tau_{0} c=100 \mathrm{fm}(\mathrm{a}), 10 \mathrm{fm}(\mathrm{b})$ and $5 \mathrm{fm}$ (c). 


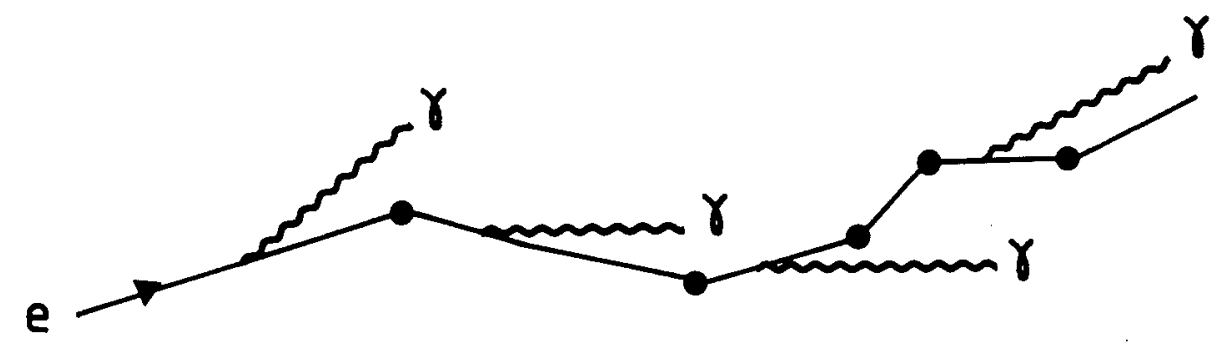




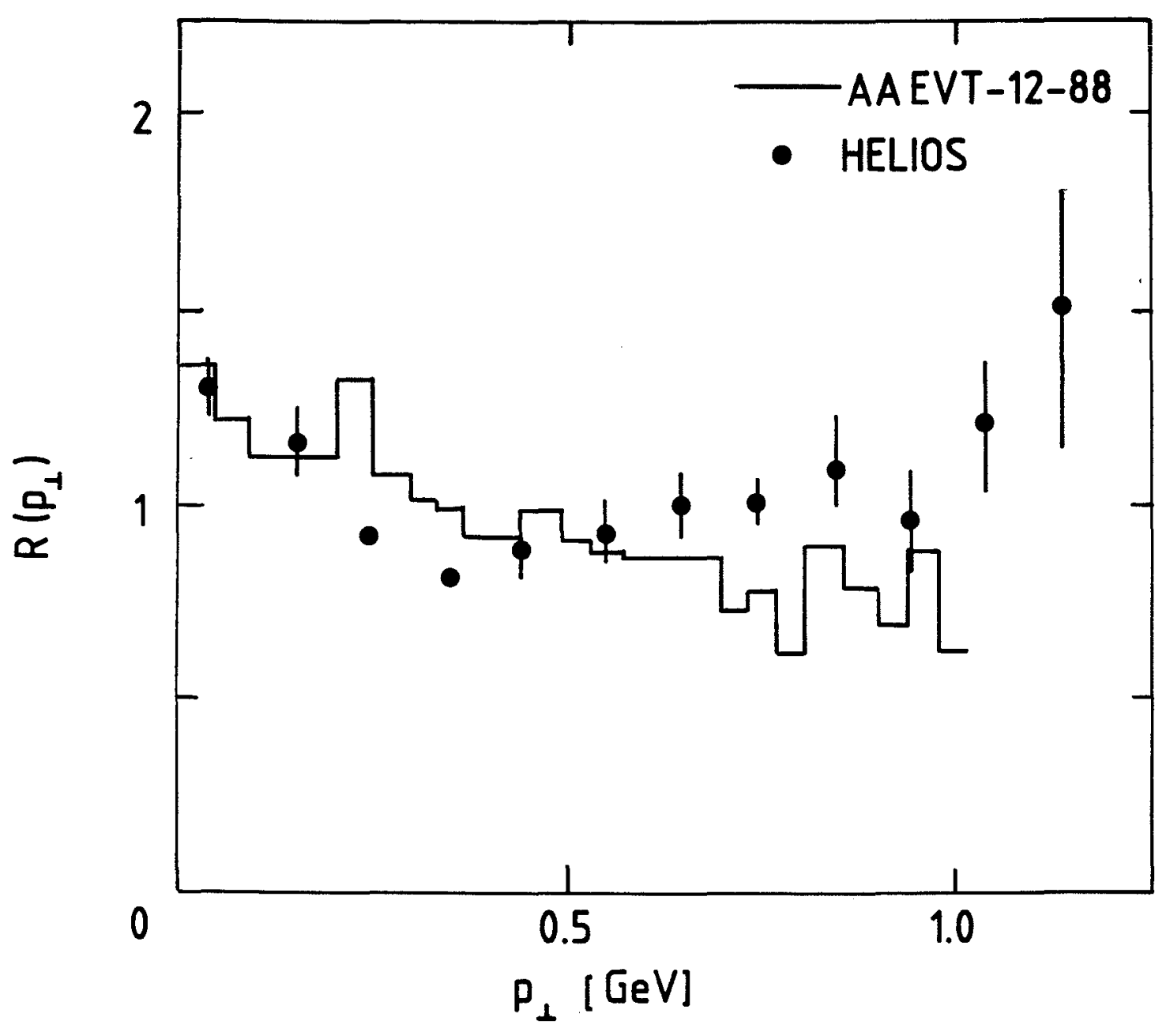




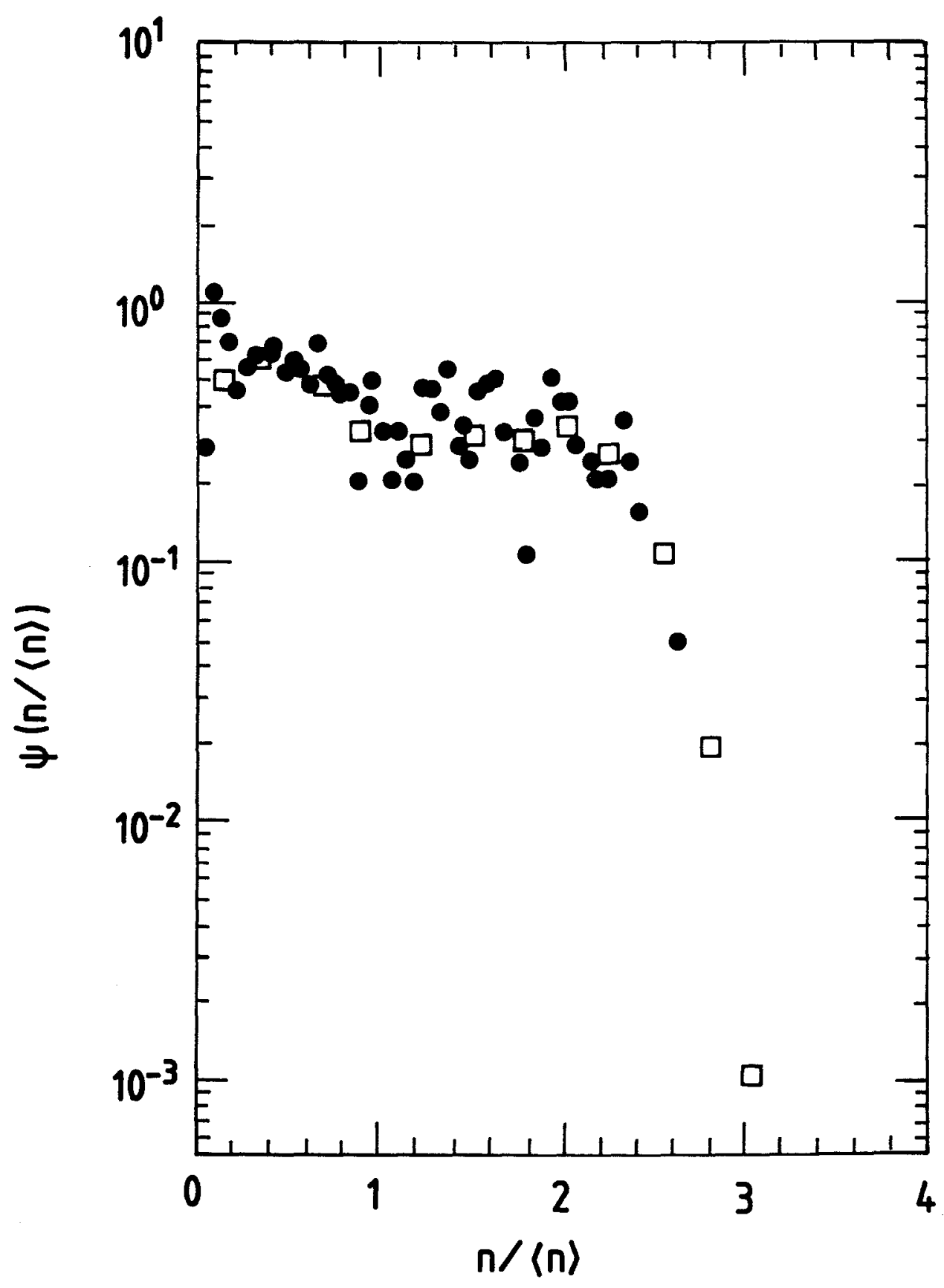




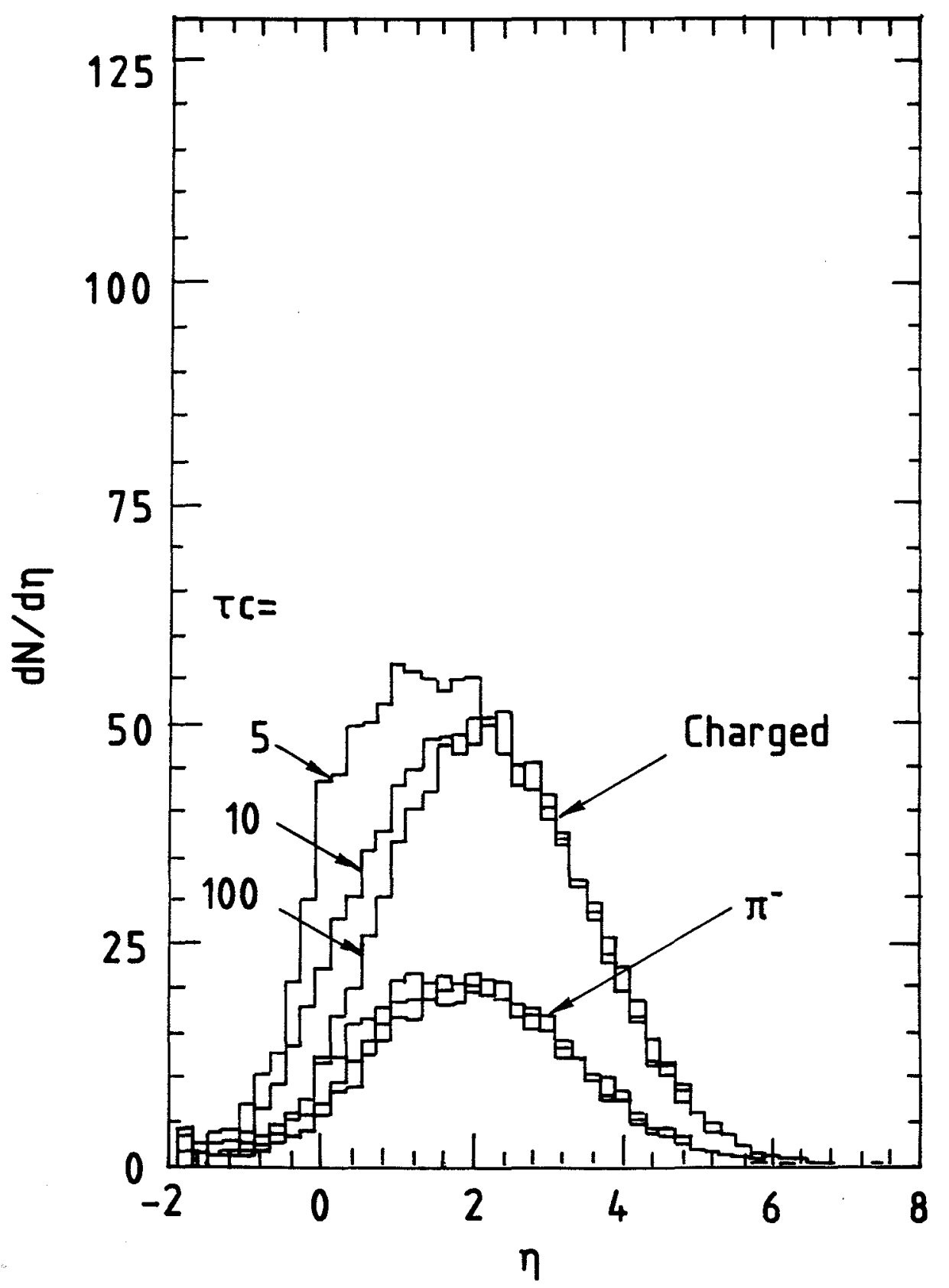




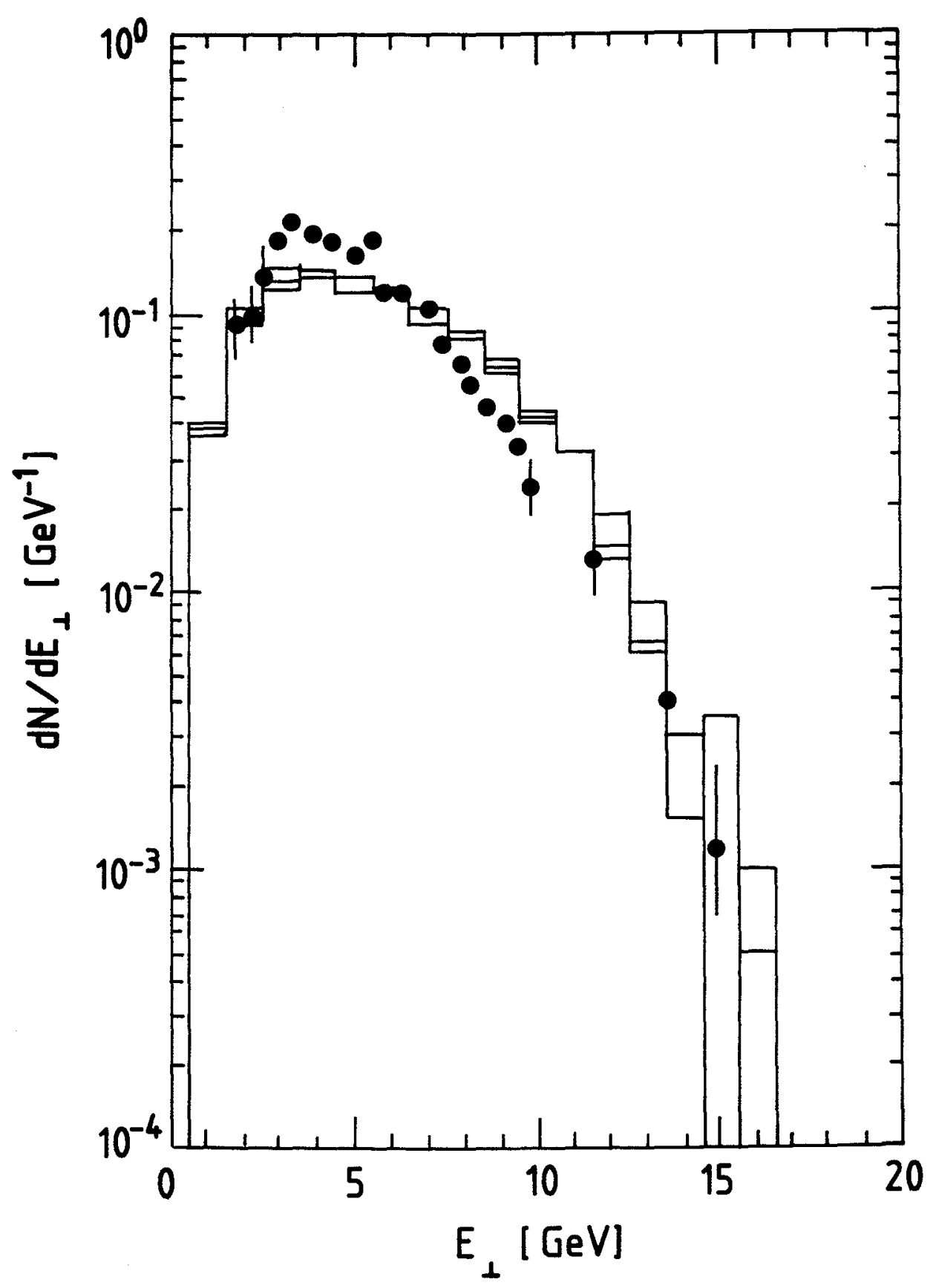




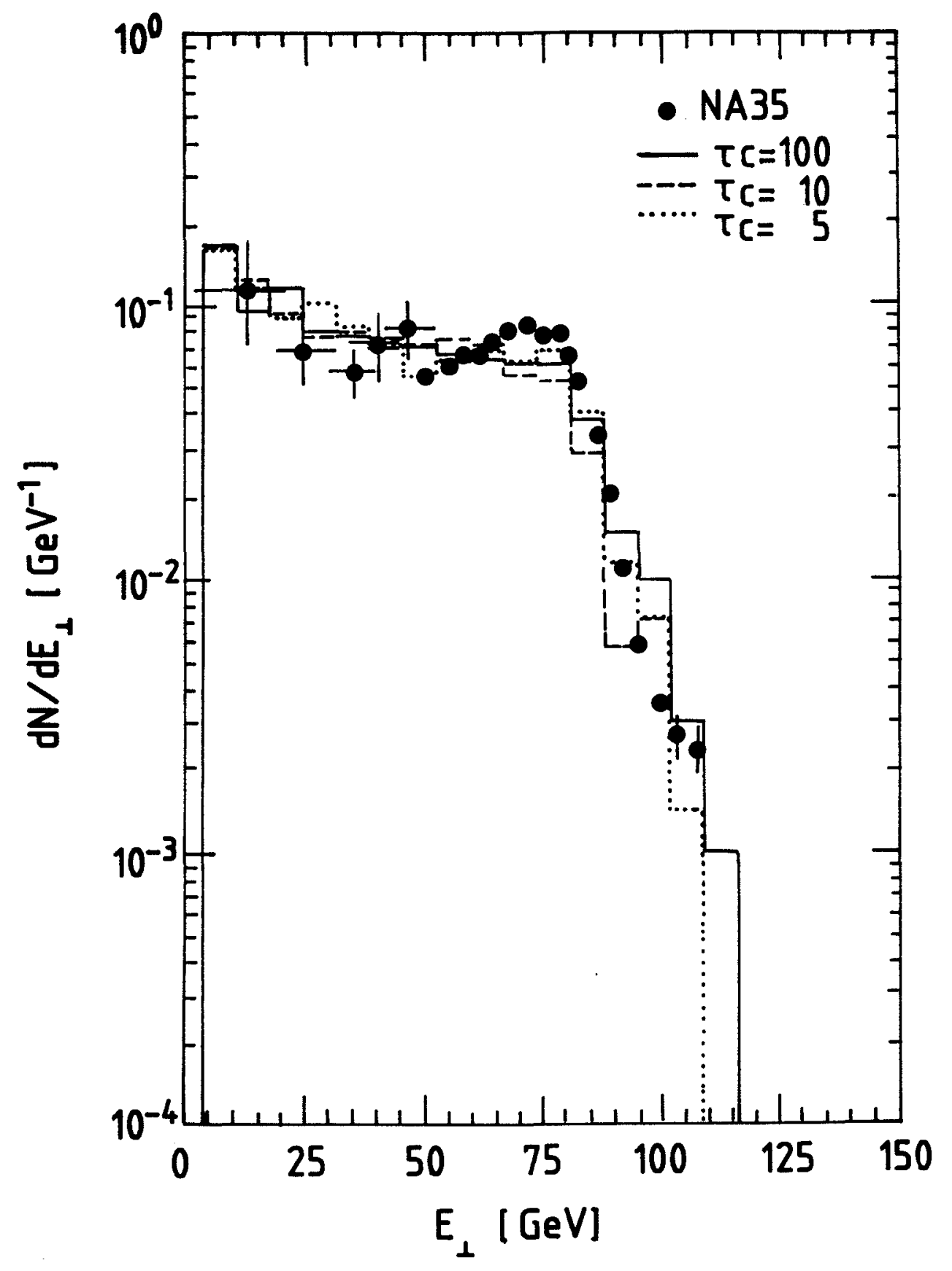




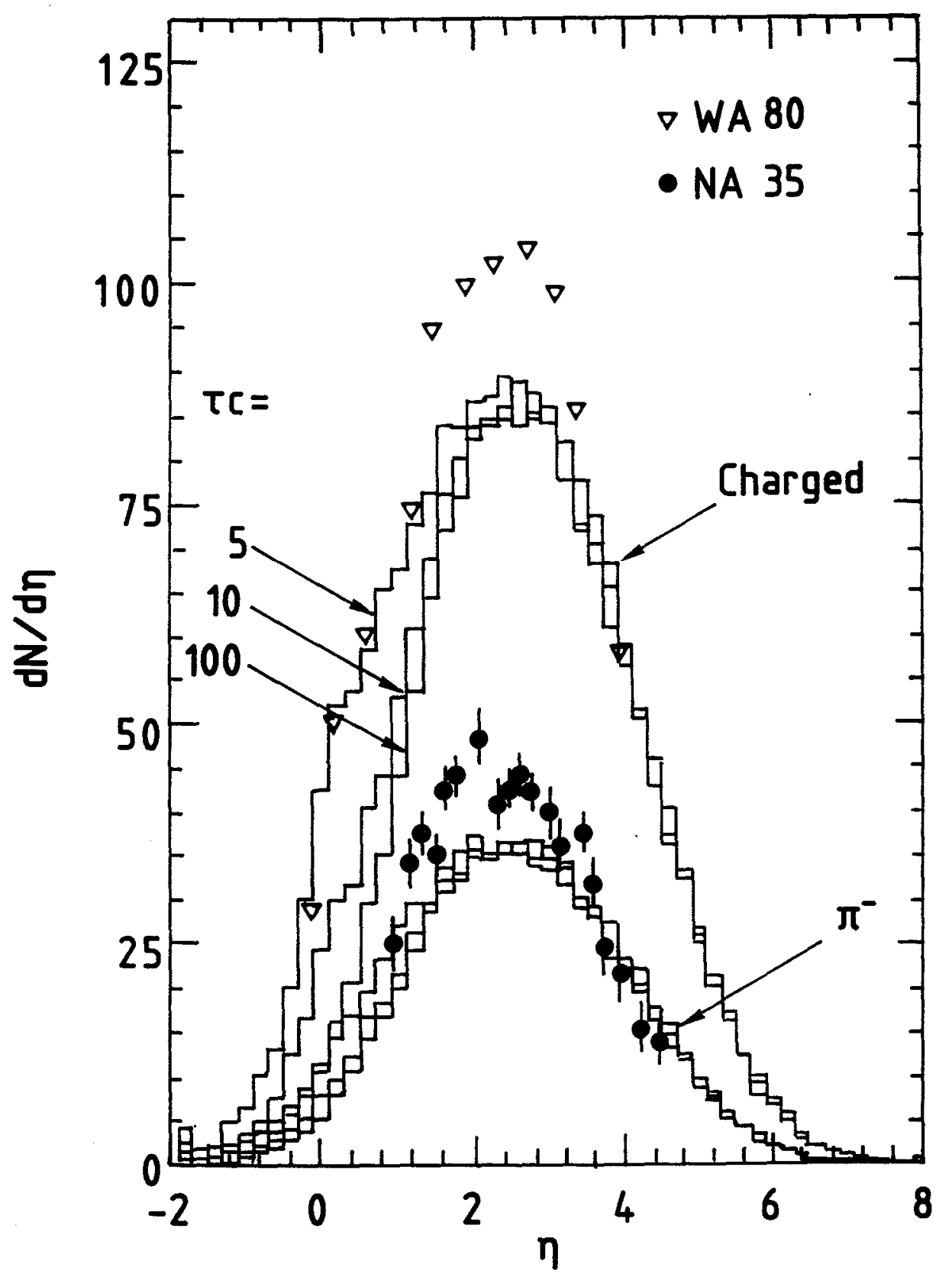




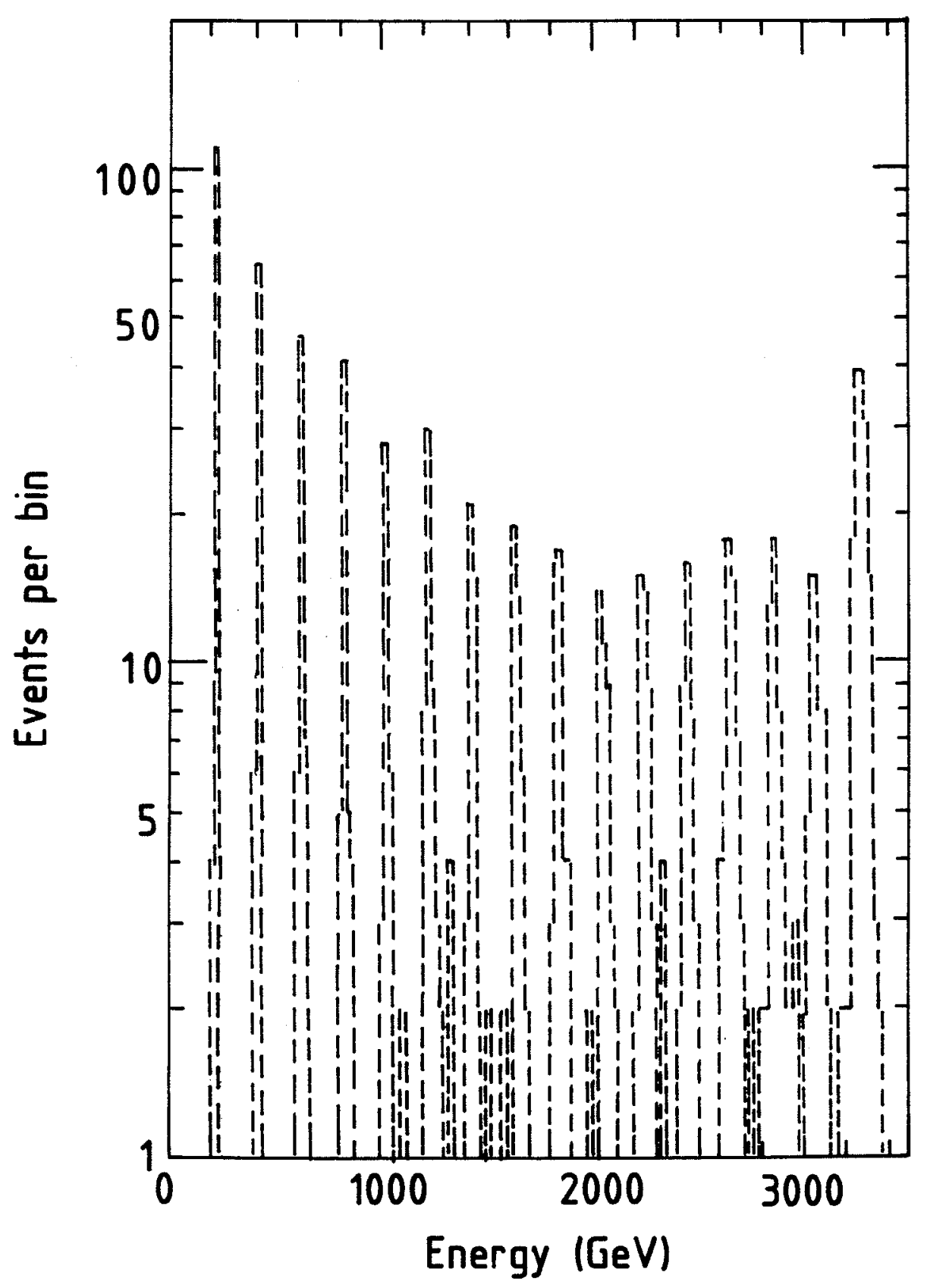




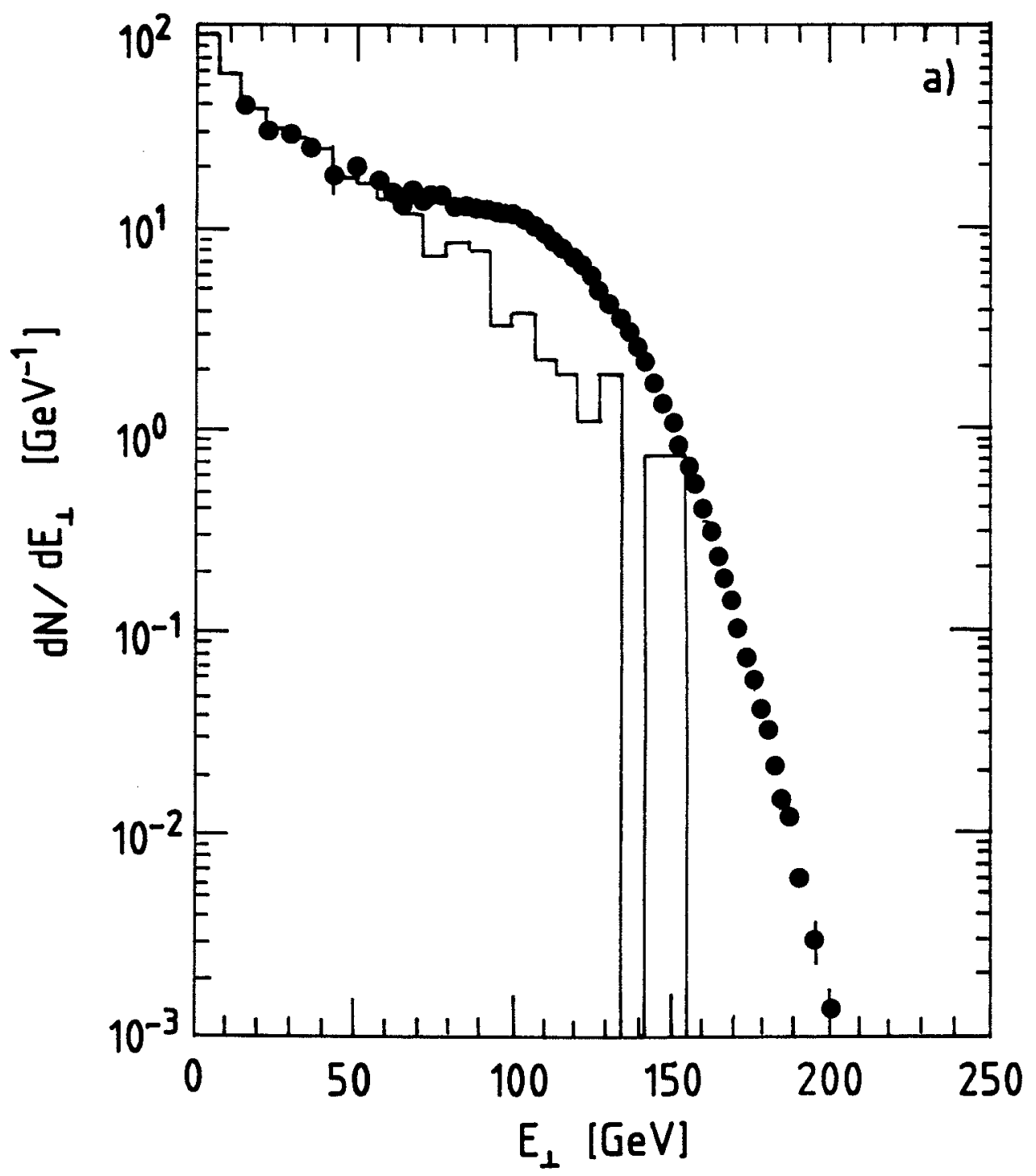




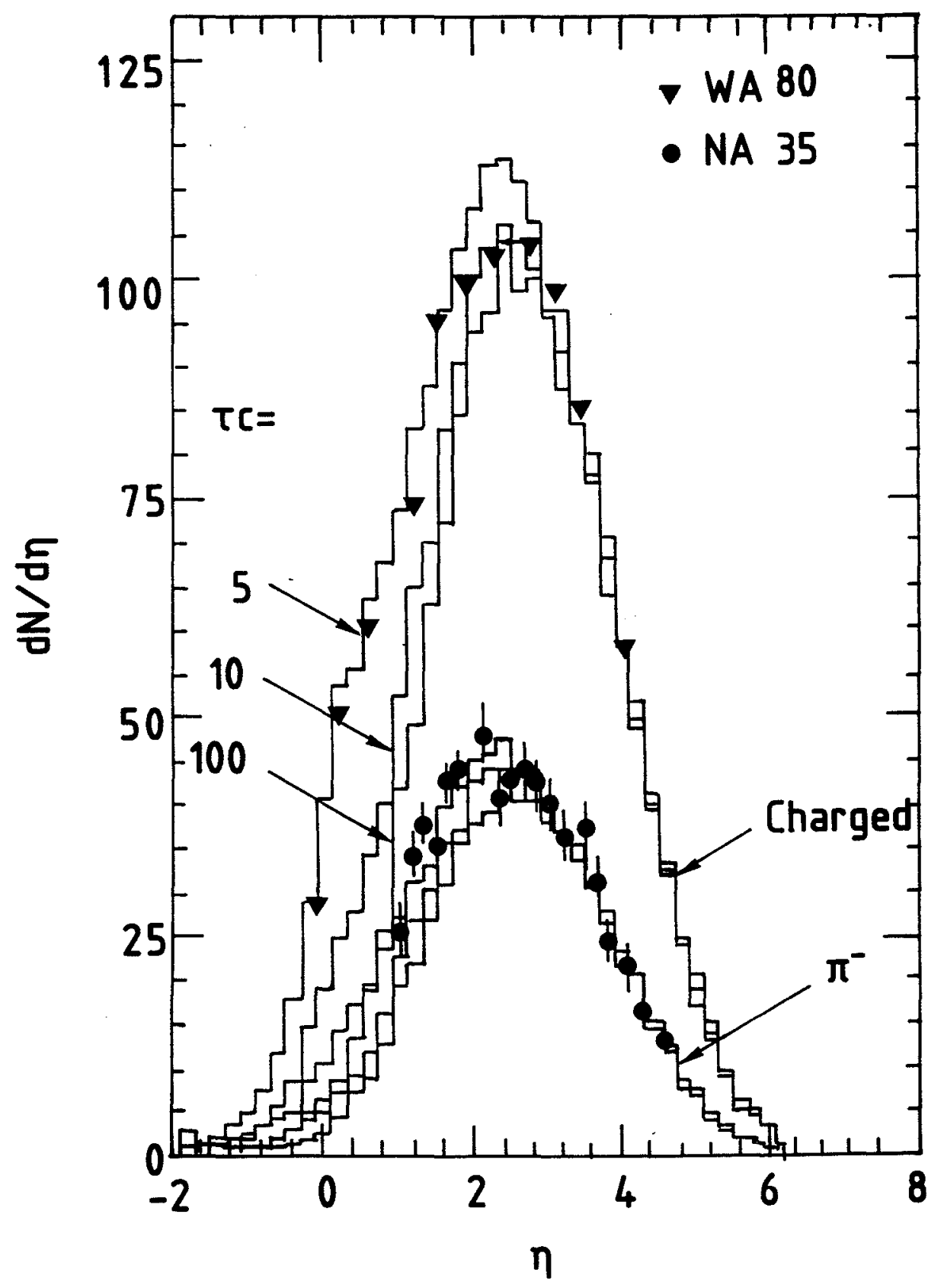




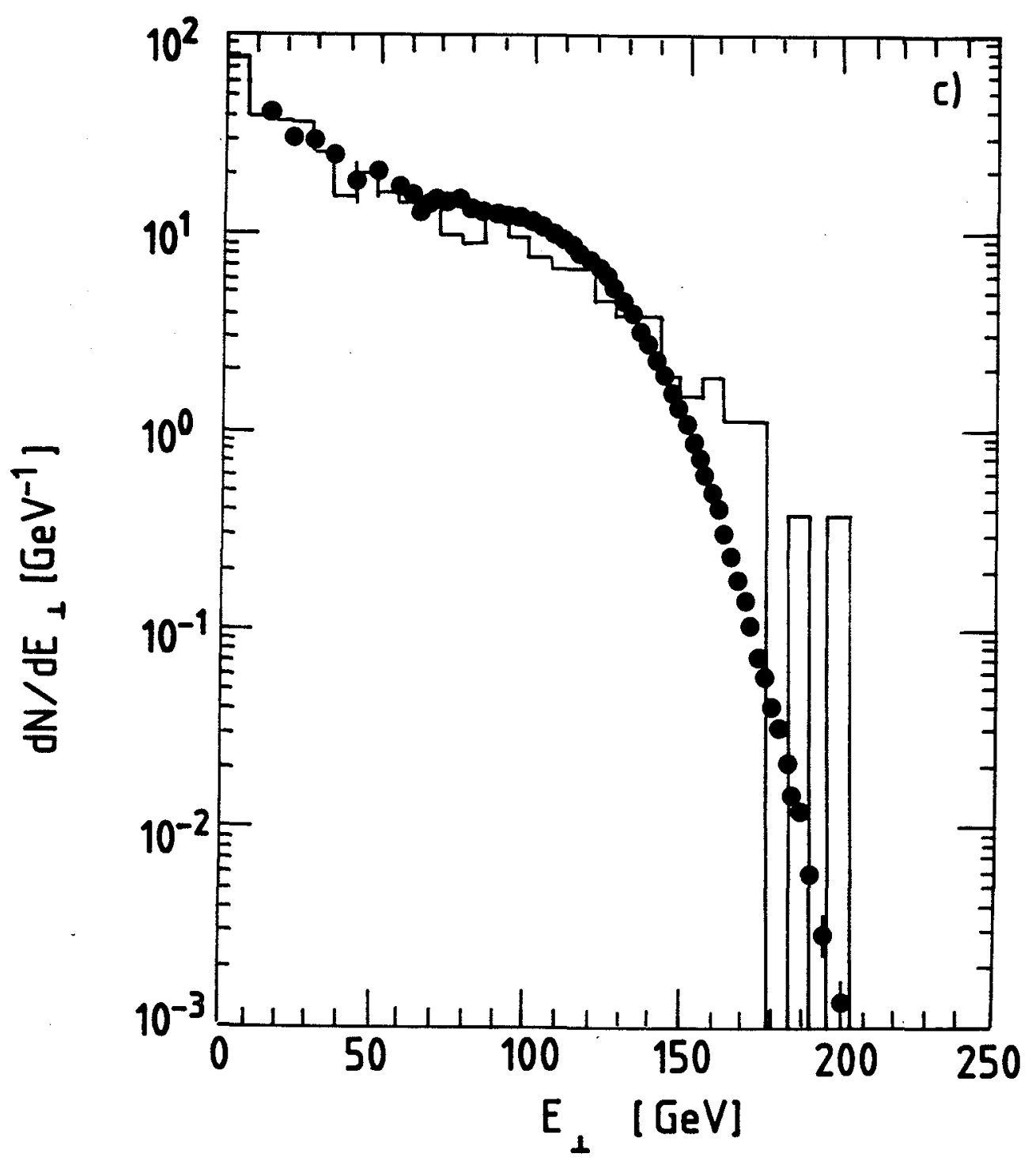




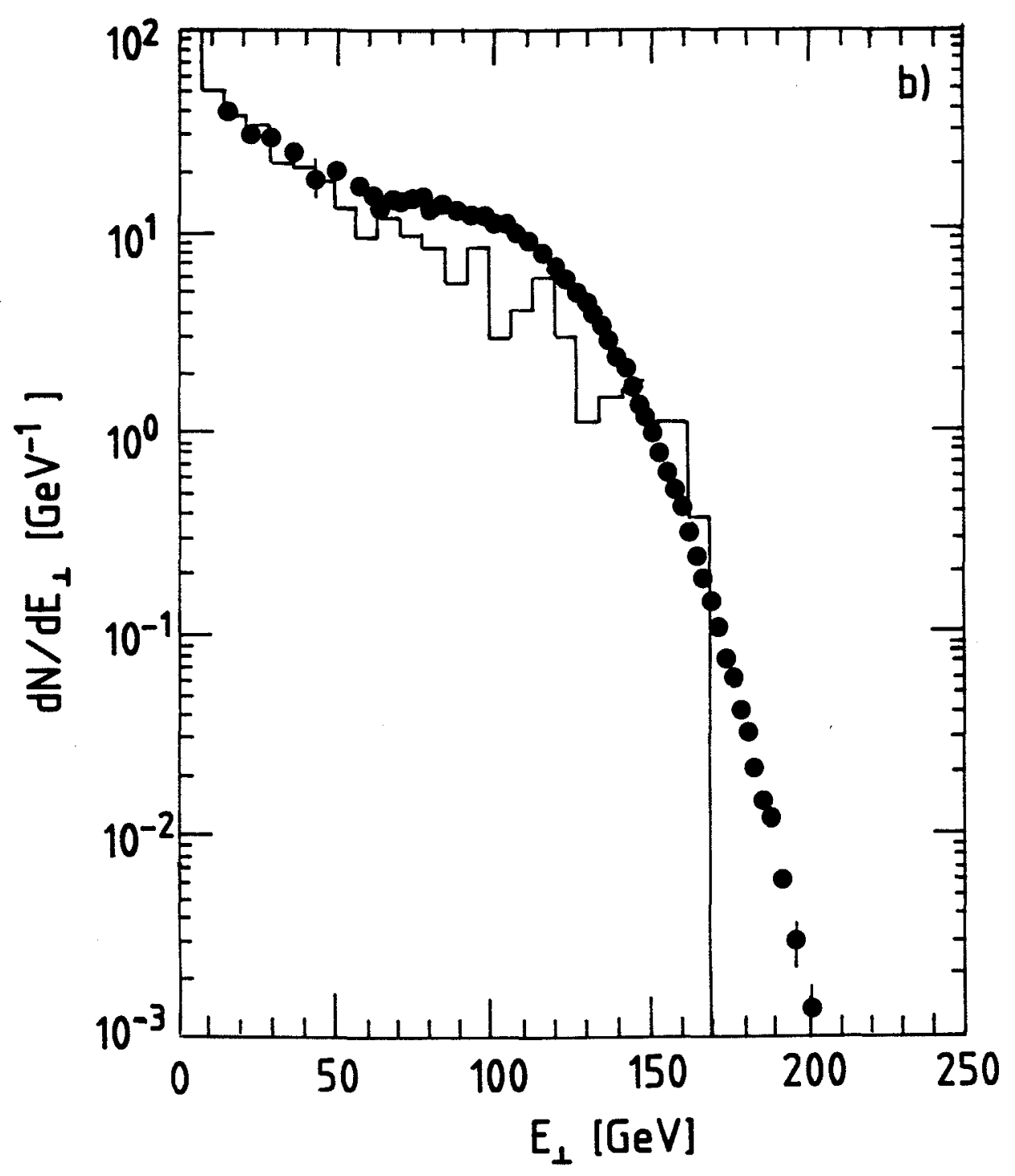




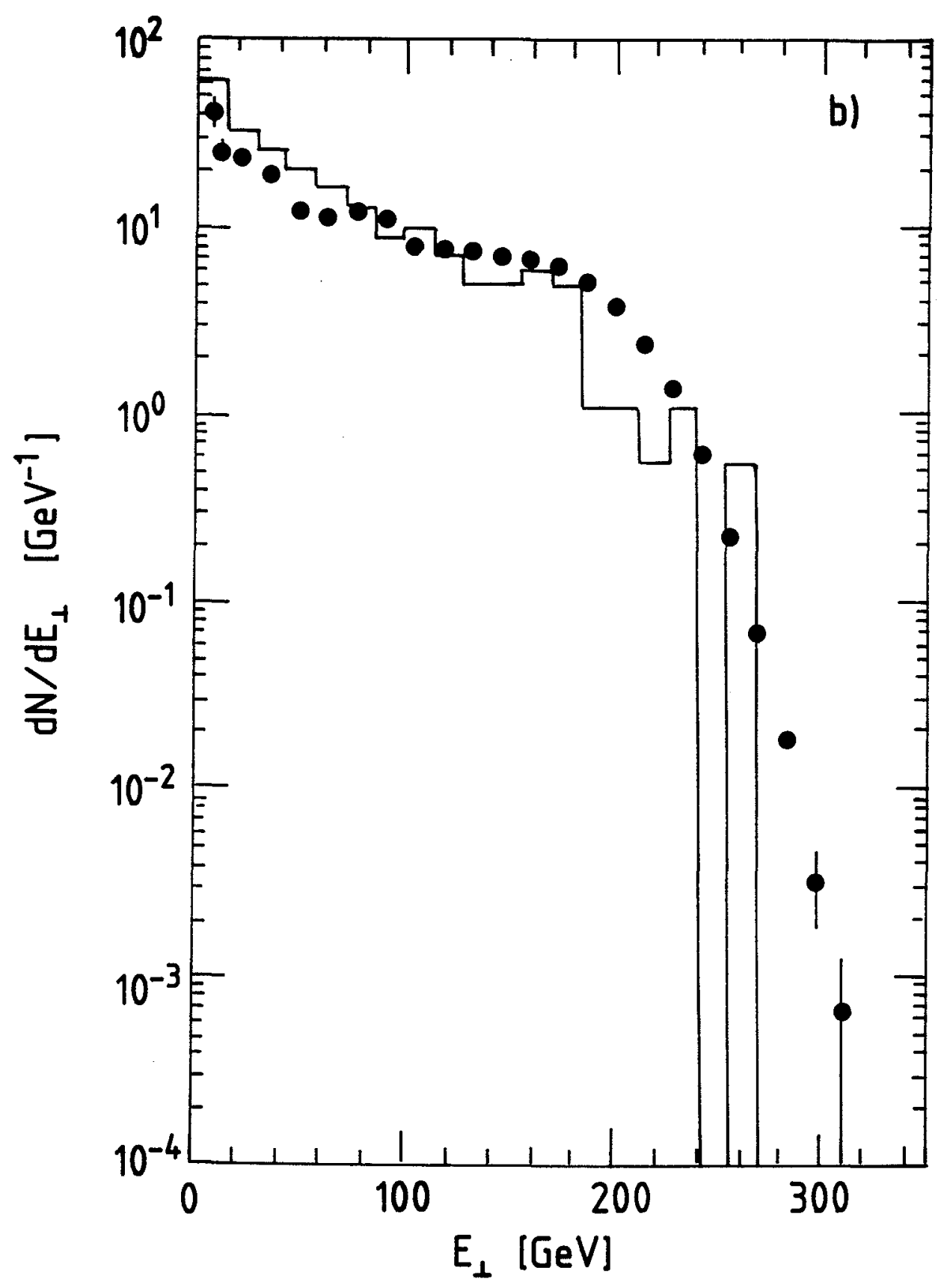




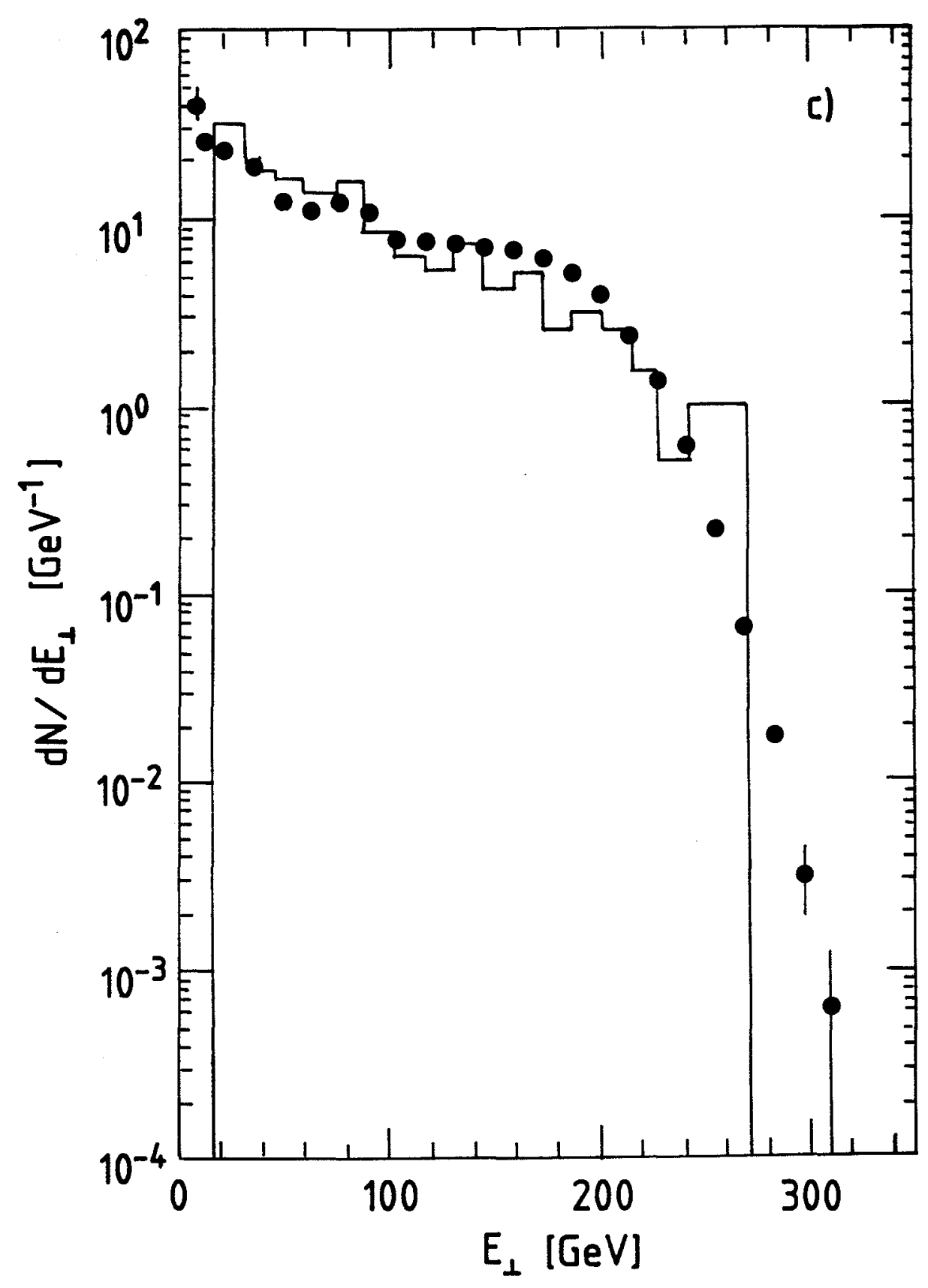




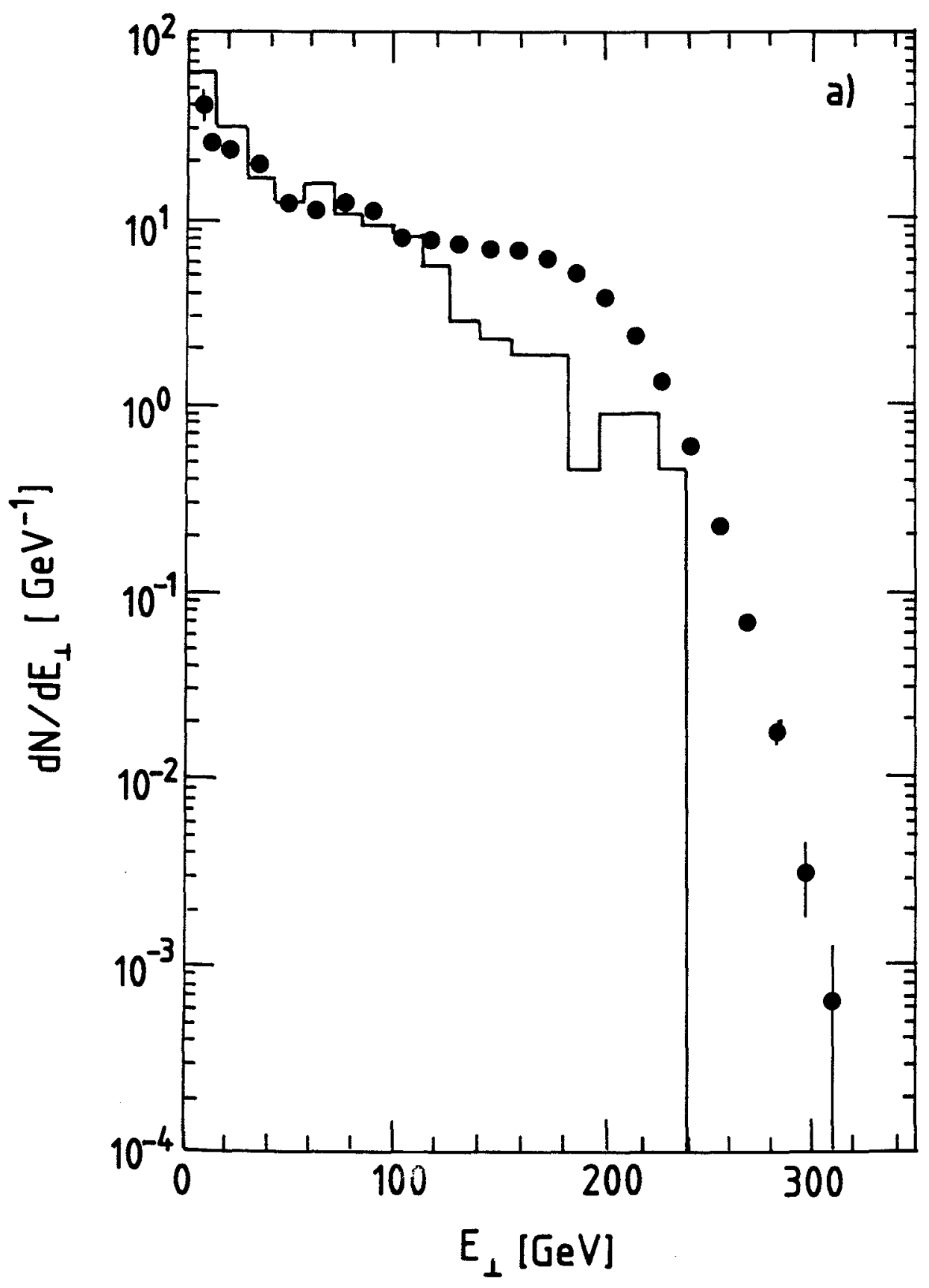

